Estudios Románicos, Volumen 29, 2020, pp. 419-431

ISSN: 0210-4911

eISSN: $1989-614 \mathrm{X}$

DOI: https://doi.org/10.6018/ER.425831

\title{
DES RÉCITS DE BATAILLE AUX IMAGES CINÉMATOGRAPHIQUES : LA DIMENSION LITTÉRAIRE DU FILM GEORGES BATAILLE, A PERTE DE VUE DE LABARTHE
}

(From Bataille tales to cinematic images: the literary dimension of the film Georges Bataille, à perte de vue by Labarthe)

\section{Mathilde Tremblais* \\ Université de Tours}

\begin{abstract}
This article looks at the literary dimension that emerges from Georges Bataille, à perte de vue, a film made by Labarthe in 1997 for the collection "Un siècle d'écrivains". It will analyze the resolutely literary narrative device put in place by Labarthe in his film. The narrative structure presented in the film will be studied and the elements that contribute to the emergence of the literary will be evoked. Labarthe proposes an approach to the fundamental concepts inherent in the fictions of Bataille, which will be the subject of reflection in order to highlight the director's work of recreating the literary universe of Bataille. The objective of this article is to show that the narrativization strategies that Labarthe deploys in his filmic narrative contribute to restoring Bataille's literality.
\end{abstract}

Keywords: Analysis of the narrative; Portrait; Narrativization; Eroticism; Fantastic; Literarity.

Résumé : Cet article s'intéresse à la dimension littéraire qui se dégage de Georges Bataille, à perte de vue, un film réalisé par Labarthe en 1997 pour la collection "Un siècle d'écrivains". Il sera question d'analyser le dispositif narratif résolument littéraire mis en place par Labarthe dans son film. La structure narrative que présente le film sera ainsi étudiée et les éléments qui contribuent à l'émergence du littéraire seront évoqués. Labarthe propose une approche des concepts

\footnotetext{
* Adresse pour la correspondance : Mathilde Tremblais, Zarandia kalea 13, 31770 Lesaka (mathilde.tremblais@, hotmail.com).
} 
fondamentaux inhérents aux fictions de Bataille qui fera l'objet d'une réflexion afin de mettre en lumière le travail de re-création de l'univers littéraire bataillien mené par le réalisateur. L'objectif de cet article est de montrer que les stratégies de narrativisation que Labarthe déploie dans son récit filmique contribuent à redonner à Bataille sa littérarité.

Mots-clés : Analyse du récit ; Portrait ; Narrativisation ; Littérarité ; Érotisme ; Fantastique.

\section{Introduction}

Le critique de cinéma, scénariste et réalisateur français André S. Labarthe (19312018) a manifesté son engagement envers la littérature de langue française à travers plusieurs de ses films, notamment Georges Bataille, à perte de vue, qu'il a réalisé en 1997 pour la collection "Un siècle d'écrivains", dirigée par Bernard Rapp. Pour cette collection, qui s'intéresse à des écrivains qui ont marqué le $\mathrm{XX}^{\mathrm{e}}$ siècle, Labarthe a orienté son regard critique vers trois auteurs qu'il affectionne tout particulièrement : Georges Bataille, Philippe Sollers et Antonin Artaud. Il consacre un film à chacun d'entre eux, le premier étant Georges Bataille, à perte de vue, où ce sont l'écrivain Georges Bataille, sa vie et ses récits qui font l'objet du portrait cinématographique. Le réalisateur s'efforce de recréer la figure de l'écrivain en s'attachant aux romans de Bataille plus qu'à ses essais, d'où la profonde littérarité qui caractérise ce film et qui motive la présente analyse.

Afin de cerner la dimension littéraire qui définit Georges Bataille, à perte de vue, il conviendra d'abord de se pencher sur la structure narrative mise en œuvre par Labarthe dans son film. La présence du littéraire sera ensuite traitée, notamment à travers l'étude du système intertextuel auquel a recours le réalisateur pour suggérer la force littéraire propre aux récits de l'écrivain. Les thèmes qui configurent l'univers de Bataille et que Labarthe choisit de mettre en scène dans son film seront évoqués, une analyse sera ainsi consacrée à l'érotisme, thème bataillien par excellence auquel le réalisateur accorde une place de premier choix. Enfin, seront interrogés les éléments qui relèvent du fantastique et qui traversent le récit de Labarthe. Les réflexions menées viseront à montrer que les dispositifs utilisés par le réalisateur pour narrativiser Georges Bataille, à perte de vue servent à mettre en valeur la puissante littérarité dont sont empreintes les œuvres de Bataille abordées dans le film.

\section{La structure narrative}

Deux voix dominent le récit de Georges Bataille, à perte de vue. Elles correspondent aux deux personnages centraux qui doivent être mentionnés d'emblée : d'une part Bataille, qui est le sujet du film et, d'autre part, un autre personnage, très discret, qui est désigné comme 'le voyageur'. Ce dernier traverse le récit, il apparaît vêtu de noir, coiffé d'un chapeau. Sa silhouette est toujours filmée de dos et, le plus souvent, en plan 
Des récits de Bataille aux images cinématographiques : la dimension littéraire du film Georges Bataille, à perte de vue de Labarthe

éloigné. Il donne l'impression d'être une sorte d'enquêteur ou de témoin qui revient sur les lieux où a vécu Bataille en quête d'une vérité sur l'auteur. Ce personnage du voyageur est incarné par le réalisateur lui-même, Labarthe. En revanche, la voix off du film qui mène la narration n'est pas celle du réalisateur mais celle du comédien JeanClaude Dauphin.

Les deux voix masculines qui dominent le récit de Georges Bataille, à perte de vue font ressortir la structure narrative complexe du film, une structure où sont mêlés différents temps. Le temps de l'énonciation correspond à 1996, année depuis laquelle s'exprime la voix off qui démarre le récit par ces mots : « Maison de Radio France, mai 1996... ». La situation de l'énonciation est mise en images par Labarthe qui filme les couloirs de la radio et un disque qui tourne, introduisant ainsi un enregistrement de Bataille daté du 10 décembre 1954. À cet instant, la voix de l'auteur surgit et le spectateur découvre la voix de Bataille, avant même de découvrir son visage ou son corps. Puis suit un flashback, un saut en arrière de plus de vingt ans qui conduit à une photographie de Bataille, un plan serré sur son visage ; il est alors âgé de trente-trois ans et vient d'écrire Histoire de l'œil (1928), il s'agit de la première allusion à l'une de ses œuvres.

Labarthe structure son récit à partir de sept enregistrements de Bataille, tous extraits de trois émissions différentes, l'une datée du 20 mai 1951, une autre du 10 décembre 1954 et la troisième de février 1961. Ces sept enregistrements sont désignés comme " trace numéro un », " trace numéro deux, « trace numéro trois », etc., et ils sont toujours accompagnés de leur date. Ces traces des enregistrements fonctionnent comme autant de marques de l'énonciation, elles sont des signes qui renvoient le spectateur à la situation d'énonciation, c'est-à-dire au studio de Radio France. Le récit de Labarthe est ainsi construit en va-et-vient entre le temps de l'énoncé et le temps de l'énonciation, 1996, l'année qui refait surface chaque fois que le réalisateur introduit un nouvel enregistrement de Bataille.

Par ailleurs, lorsque l'énigmatique voyageur fait irruption dans le récit, l'année 1996 est à nouveau citée, la voix off indique que « le 26 avril 1996, un voyageur et son ombre arrivent à Vézelay », le lieu symbolique où s'installa Bataille en 1943, ou encore la voix off informe que « mardi 23 avril 1996, le voyageur se rend à Orléans ». Les indications temporelles très précises des enregistrements radiophoniques de Bataille ou des déplacements du voyageur sur les lieux où a vécu l'auteur servent à ancrer le film dans le réel; de même les événements historiques attestés sont autant de procédés que le réalisateur met en œuvre pour montrer la véracité discursive de son film.

Dans Georges Bataille, à perte de vue la voix off et la voix de Bataille se complètent et se répondent, elles créent une sorte de dialogisme, de polyphonie narrative. En outre, les enregistrements de Bataille opèrent comme un lieu de témoignage qui permet au spectateur de découvrir l'intimité de la parole de l'écrivain. Ainsi, dans sa narration, Labarthe met-il en place un espace scénique où la voix de Bataille surgit, retentit et résonne. Le cinéaste libère la parole de l'écrivain, il parvient à donner vie à la voix fascinante et à l'élocution si particulière de Bataille, des traits qui ont frappé tous ceux qui l'ont connu : 
Lente a toujours été, par exemple, son élocution, lente et circonspecte, soucieuse de la minutie du détail. Tout au plus, avec les années, a-t-elle pu devenir plus lente ; la voix est belle et tous le disent : le timbre de la voix - légèrement tremblé - ajouté à une élocution si particulière sont pour beaucoup dans son évidente séduction (Surya 1992 : 473).

Pour ce qui est des principaux événements de la vie de Bataille, Labarthe y fait référence en suivant une ligne chronologique, en respectant l'ordre des passages obligés de toute biographie. Le réalisateur commence ainsi par l'enfance et utilise un deuxième enregistrement de Bataille, celui-ci daté du 20 mai 1951, dans lequel l'auteur évoque l'histoire de son père, de ce père aveugle et paralytique qui l'a profondément marqué. Quand cet enregistrement s'achève, Labarthe prolonge l'histoire du père en choisissant de raconter les conditions dans lesquelles celui-ci trouva la mort, abandonné des siens, durant la première guerre mondiale. Labarthe illustre cet épisode avec des images d'archives de la guerre et ponctue ce récit par une citation de Bataille : " j'ai abandonné seul, mon père, l'aveugle, le paralytique, le fou, criant et gesticulant de douleur, cloué dans un fauteuil crevé » (Bataille 1973 : 504). Le réalisateur retrace les principaux événements de la vie de Bataille en insistant sur la mort de ses êtres chers : après la mort du père, le réalisateur fait référence à la mort de la mère de Bataille, le 15 janvier 1930, et à la mort de sa compagne, Laure, en novembre 1938, alors qu'elle n'était âgée que de trente-cinq ans. Enfin, au sujet de la structure narrative il convient d'ajouter que Labarthe dégage aussi les jalons de la carrière de Bataille de manière chronologique, en commençant par 1928 et Histoire de l'œil, suit l'année 1929 avec la création de la revue Documents, puis la création d'Acéphale en 1936 et le réalisateur s'étend jusqu'à 1957, date où paraît Le Bleu du ciel. Labarthe confère ainsi au littéraire un espace qui s'avère primordial, aussi bien en dévoilant le pouvoir de suggestion des récits qu'en racontant l'engagement de Bataille en tant qu'homme de lettres et la place marginale qu'il occupait dans le paysage littéraire et intellectuel de son époque.

\section{La présence du littéraire}

Le film Georges Bataille, à perte de vue est traversé de citations de l'œuvre de Bataille, comme celle précédemment mentionnée. Précisons que cette citation qui évoque la mort du père est extraite du texte Le Coupable (1944) mais Labarthe choisit de ne pas donner le titre de ce récit. Quand le réalisateur cite le titre d'une œuvre, il en montre les pages à l'écran, mais dans d'autres cas, comme dans celui auquel nous venons de faire référence, le réalisateur préfère ne pas donner le titre du texte auquel appartient la citation, peut-être pour inviter le lecteur averti de l'œuvre bataillienne à deviner de quel récit la citation est extraite. Par ces procédés, l'explicite du citationnel ou l'implicite de l'allusif, Labarthe met en place un système intertextuel particulièrement intéressant, un espace où les fictions de Bataille dialoguent entre elles pour permettre au littéraire et au poétique d'émerger du texte qui sous-tend Georges Bataille, à perte de vue. 
Des récits de Bataille aux images cinématographiques : la dimension littéraire du film Georges Bataille, à perte de vue de Labarthe

Par ailleurs, Labarthe parvient à rendre le littéraire présent en faisant très souvent apparaître les écrits à l'écran, tout d'abord la première œuvre que Bataille composa en 1918, Notre-Dame de Reims, un texte que Michel Surya, dans la présentation de Discussion sur le péché, caractérise de " livre au plus haut point chrétien » (Surya 2010 : 13). Vient ensuite Histoire de l'ail, la voix off en lit un passage et les lignes des pages du livre sont filmées, ainsi que l'un des dessins de Masson illustrant l'histoire. Le fait de filmer les caractères des pages et de lire l'extrait à voix haute renforce l'intérêt que suscite le contenu du texte et accentue sa portée littéraire.

Quand il arrive à Vézelay le 26 avril 1996, le voyageur feuillette les cahiers de Bataille. Labarthe permet au spectateur de pénétrer dans l'intimité de l'auteur en filmant son écriture, ainsi que les ratures, les modifications ou les ajouts propres à une œuvre en élaboration ou en construction. Le réalisateur montre les manuscrits, les pages du journal intime de Bataille, des fragments qui deviendront ses futures œuvres. Il fait référence à l'autobiographie dont Bataille avait eu le projet mais qu'il ne mena pas à terme, l'inachèvement ${ }^{1}$ étant un trait distinctif de nombreux textes de l'écrivain.

La présence du littéraire est aussi palpable à travers certaines querelles entre écrivains auxquelles Labarthe choisit de faire allusion. Le réalisateur évoque ainsi les animosités qu'a suscitées Bataille chez ses contemporains, notamment chez Breton qui voyait en l'auteur d'Histoire de l'œil un obsédé qui ne considère au monde que « ce qu'il y a de plus vil, de plus décourageant et de plus corrompu » (Breton 1929 : 144). Breton n'était pourtant pas le plus féroce des ennemis de Bataille, Boris Souvarine traitait celui-ci de « détraqué sexuel » et le faisait passer pour un homme hautement pervers, au « comportement pathologique » (Souvarine 1983 : 19-22).

Labarthe aborde la revue Critique, créée par Bataille en 1946 et pour laquelle il rédigea de nombreux articles ${ }^{2}$ de critique littéraire jusqu'en 1961, soit un an avant sa mort. Quand Labarthe fait référence à cette revue grâce à laquelle Bataille souhaitait représenter l'essentiel de la pensée humaine, le cinéaste souligne l'isolement auquel était contraint l'écrivain et aussi le fait que ses contemporains n'étaient pas sensibles à l'approfondissement de sa pensée. Dans ce sens, à la fin du film, la voix off éclaire : « comme toujours, la douloureuse liberté de sa pensée s'accompagne d'un effort de conceptualisation qui sera longtemps incompris : je ne suis pas un philosophe précise-t-il

1 Dans ce sens, Michel Surya fait remarquer à propos de l'œuvre de Georges Bataille : « Rien en elle de stable ni de statique ; rien qui ait le caractère de l'achèvement » (Surya 1997, XIV). À ce sujet, Denis Hollier apporte la précision suivante : «l'inachèvement doit toujours être pensé, à propos des textes de Bataille, comme l'un des gestes constitutifs de son écriture, jamais comme un simple accident » (Hollier 1974 : 287). Labarthe, à la fin de son film, évoque, lui aussi, l'inachèvement propre aux projets littéraires de Bataille quand la voix off souligne que « l'ironie noire qui a baptisé CEuvres complètes un ensemble de textes dont chaque page célèbre le triomphe de l'inachevé ».

2 En effet, pour Critique qu'il créa et dirigea de 1946 à1962, Bataille écrivit de nombreux articles sur de prestigieux auteurs tels que Miller, Proust, Baudelaire ou Sade. De même, il s'intéressa à ses contemporains : Prévert, Camus, Char ou Genet. Tous les articles que Bataille rédigea pour Critique figurent dans les tomes XI et XII des CEuvres Complètes, nous avons compté quatre-vingt-trois articles, qui représentent en tout sept cent quatrevingt-treize pages, une somme qui reflète l'engagement que manifestait Bataille pour la littérature. 
pourtant, je suis un saint, peut-être un fou ${ }^{3} »$. Labarthe insiste sur l'isolement dans lequel était plongé Bataille, lui qui, rappelons-le, s'était refusé à intégrer deux des principaux mouvements qui marquèrent son temps : il s'était montré clairement hostile au surréalisme depuis ses débuts et il n'avait pas non plus voulu adhérer à l'existentialisme de Sartre. Les spécialistes de Bataille n'ont pas manqué de faire remarquer le mépris que Bataille éveillait chez ses contemporains, par exemple Gilles Mayné déclare dans son essai Georges Bataille, l'érotisme et l'écriture : "l'écrivain était souvent dénigré comme pornographe ou comme indigne du rang de philosophe par des figures aussi reconnues que Breton et Sartre » (Mayné 2003 : 328). Le manque d'académisme de Bataille ou le fait qu'il ne soit pas un penseur très systématiste ont pu contribuer au rejet dont il était l'objet. Peut-être la position d'isolement dans laquelle se trouvait Bataille était-elle due au fait que, comme le dit Michel Surya : "Cet homme était fait pour dire des choses qu'en principe on fuit, toute son œuvre dit des choses que tous fuient» (Surya 1997 : XI).

En plus des textes de Bataille qui sont cités et lus et des personnes qui interviennent dans le film pour parler de l'écrivain, comme Pierre Klossowski, et en plus des controverses littéraires évoquées, le littéraire est présent à travers l'importance qu'accorde Labarthe à la bibliothèque d'Orléans, dont Bataille a été le conservateur à partir de 1951. Dans son livre Bataille conservateur. Emprunts intimes d'un bibliothécaire, Jean-Louis Cornille écrit que Bataille a été « un gestionnaire officiel de la mémoire collective, un professionnel assermenté du livre » (Cornille 2004 : 7). Dans le film de Labarthe, le voyageur se rend sur les lieux de la Bibliothèque d'Orléans et y découvre la salle de lecture. Le réalisateur accompagne les images du lieu de l'enregistrement d'un entretien qu'accorde l'écrivain à Madeleine Chapsal, qui était à l'époque une jeune journaliste. Cet entretien a eu lieu au printemps 1961, quelques mois seulement avant la mort de Bataille ; il est publié dans le livre de Madeleine Chapsal, Les écrivains en personne (1973). Dans cette séquence, comme dans l'ensemble du film, l'écoute de la parole enregistrée de Bataille et la présence de photographies de l'écrivain reflètent le travail mené par Labarthe afin de mettre en lumière la singularité de son personnage, un travail propre au portrait dont l'entreprise est ici pertinemment décrite :

En tant que mode d'exposition de la singularité d'un individu, le portrait est l'aboutissement d'un travail d'appropriation de cette singularité par un auteur. Il se veut expression personnelle tout en se gardant de l'écueil du subjectif. Il ne se cherche pas seulement de l'intérieur de l'expérience racontée mais de l'intérieur des paroles enregistrées, des gestes retenus, des comportements observés. Autrement dit, le portrait comporte toujours quelque chose qui appartient à son auteur/chercheur. Il est plein du regard que le chercheur porte sur son personnage et qu'il partage avec le lecteur (l'idée de personnage plutôt que de personne ou d'individu nous permet de le rapprocher davantage de la démarche du cinéaste). Au-delà de la description de la situation de communication et de la reproduction des témoignages selon un certain agencement, le portrait est d'abord un texte porté par un auteur (Rueda $2014: 180$ ).

3 Dans Méthode de méditation (1947b), Bataille déclare avec une certaine ironie ou provocation : « Je ne suis pas un philosophe mais un saint, peut-être un fou » (Bataille 1973 : 218). 
Des récits de Bataille aux images cinématographiques : la dimension littéraire du film Georges Bataille, à perte de vue de Labarthe

Labarthe se fait le garant de la parole de Bataille et la réinvestit, en élaborant un portrait qui laisse aisément deviner le regard qu'il porte sur son personnage, l'admiration illimitée et la complicité intellectuelle qu'il éprouve envers l'écrivain Bataille. En offrant un portrait qui dépasse les limites du registre purement biographique, le cinéaste parvient à recréer la figure de l'auteur et à mettre en images les principaux thèmes qui hantent la pensée de Bataille et qui peuplent ses œuvres fictionnelles.

\section{La re-création de l'univers littéraire bataillien}

Dans son film, Labarthe fait référence aux principaux concepts qui structurent le système de pensée de Bataille, ainsi qu'aux sujets qui configurent son univers littéraire. Il le fait parfois au moyen d'une citation de l'écrivain, par exemple celle-ci : " Le monde n'est habitable qu'à la condition que rien n'y soit respecté ». À travers cette citation de Bataille, Labarthe introduit le thème de l'irrespect, un thème fondamental de la pensée bataillienne et sur lequel plusieurs spécialistes ont réfléchi, comme JeanMichel Besnier dans Éloge de l'irrespect (1998). En rapport avec l'irrespect, dans le film de Labarthe le verbe 'renverser' apparaît à maintes reprises pour décrire l'élan propre à Bataille : «renverser le sens du malheur », « renverser le ciel ou l'assignation d'un but au monde ». Ces mots font écho aux nombreux renversements qui traversent les textes de Bataille, comme dans la préface de Madame Edwarda (1941) où l'auteur prône " un entier renversement » (Bataille 2010a : 13). L'idée de renverser est au cœur de la philosophie de Bataille qui vise à bousculer le système et l'ordre établi. N'oublions pas que Bataille était à son époque celui qui ébranlait l'ensemble des discours, lui qui très jeune voulait « s'opposer comme une brute à tout système " (Bataille 1970a : 183).

D'autres thèmes batailliens sont cités comme l'angoisse, l'impossible ou encore la chance. Labarthe s'attarde davantage sur le rire qui, rappelons-le, a été pour Bataille une découverte décisive quand il rencontra Bergson, à Londres, en 1930. L'écrivain ressentit tout au long de sa vie la signification tragique du rire. Labarthe montre une photo de Bataille où il rit, d'un rire qui laisse généreusement voir sa dentition. D'ailleurs l'auteur devait avoir un rire assez surprenant et même une dentition particulière, si l'on en croit son ami Michel Leiris qui écrit au sujet de Bataille : "À ses yeux assez rapprochés et enfoncés, riches de tout Le bleu du ciel, s'alliait sa curieuse dentition de bête des bois " (Leiris 1963 : 685).

Labarthe accorde dans son film une place déterminante à la religion, il ne faut pas oublier que Bataille, pendant plusieurs années, a caressé le projet de se consacrer à Dieu. Il évoque la foi de Bataille, le séminaire de Saint Flour, puis la perte radicale de la foi. La mort est omniprésente dans le film de Labarthe, non seulement à travers l'allusion aux êtres que l'écrivain a perdus mais aussi à travers sa propre mort que Bataille ne cesse d'envisager. Dans sa biographie, Michel Surya soutient à ce propos : « Bataille mâche et remâche sa mort ; il vit comme il est rare qu'on vive : avec » (Surya 1992 : 191). Bataille lui-même écrivait : « ma propre mort m'obsède encore comme une cochonnerie obscène, et par conséquent horriblement désirable » (Bataille 1970b : 87). 
Le mal est un thème bataillien qui, comme tel, n'est pas cité par Labarthe, mais est suggéré à travers d'autres éléments présents dans le film. Michel Surya, dans sa biographie, affirme que "Bataille aurait été, et de longtemps, doublement et contradictoirement attiré par le bien et le mal » (Surya 1992: 521). Soulignons que le mot 'contradictions', ou bien un terme proche, est celui qui revient le plus sous la plume de tous les portraitistes de Bataille. Dans ce sens, Jean Piel dit de l'écrivain qu'il est «l'homme où s'affrontent les contraires » (Piel 1982:145) et Michel Surya rappelle, comme s'il s'agissait d'une prédestination, que «Bataille est né dans un petit village roman, entre l'église et la porte des Boucheries » (1992: 420). De même, le biographe de Bataille soutient que « le bordel et le cloître semblent avoir fait l'oscillation de Bataille toute sa vie » (1992: 538) ou encore que Bataille était " dévot en 1922, débauché en $1924 »$ (1992 : 72). Un plan, à la douzième minute de Georges Bataille, à perte de vue, a peut-être le pouvoir de suggérer ce choc des contraires qui déchire l'écrivain au plus profond de son être. En effet, Labarthe réunit dans un même plan, filmé en contreplongée, le clocher d'une église et la façade d'un café dont les lumières scintillent, deux lieux très opposés qui évoquent l'esprit religieux et la débauche qui ont caractérisé la vie de l'auteur.

Concernant la débauche, la voix off du film explique : «Dans la vie de débauche que mène Bataille et où il puise ses interrogations les plus fortes... ». Elle rejoint ainsi les mots du narrateur d'Histoire de l'œil : " La débauche que je connais souille non seulement mon corps et mes pensées mais tout ce que j'imagine devant elle et surtout l'univers étoilé » (Bataille 2001a : 63). La débauche chez Bataille est souvent signifiée à travers le bordel, un lieu qui était cher à l'écrivain, selon les dires de ses amis Michel Leiris et Jean Piel. Michel Leiris désigne ainsi Bataille comme « ce mystique de la débauche » (Leiris $1988: 12$ ) et comme un « habitué des tripots et de la compagnie des prostituées comme tant de héros de la littérature russe » (1988:23). Dans son ouvrage Pour Bataille. Etre, chance, souveraineté, Bernard Sichère écrit que "Bataille fut un homme aimé des femmes, un homme à femmes, un homme de la vie intense et des bordels » (Sichère 2006 : 104).

Dans Le Coupable, Bataille écrit : « Une maison close est ma véritable église, la seule assez inapaisante » (Bataille 1973 : 247). Labarthe filme quelques pages de l'œuvre et il accompagne les images des pages griffonnées par la plume de Bataille de la voix de l'écrivain qui avoue, dans l'enregistrement trace numéro cinq, que Le Coupable est : « le livre dans lequel je suis le plus moi-même, celui qui me ressemble le plus ». Cette confession de Bataille intervient juste avant la séquence dont Madame Edwarda fait l'objet dans le film et qui a pour décor un bordel, le lieu par excellence de l'érotisme bataillien.

\section{La notion de l'érotisme}

Au sujet de l'érotisme, il faut rappeler que Bataille était, dans les années cinquante, l'un des grands maîtres à penser de l'érotisme, une autorité indiscutable en la matière. Le premier texte érotique que Labarthe cite est extrait d'Histoire de l'œil, il s'agit de 
Des récits de Bataille aux images cinématographiques : la dimension littéraire du film Georges Bataille, à perte de vue de Labarthe

l'épisode au cours duquel Simone introduit dans l'antre de sa féminité l'œil du prêtre Don Aminado. Le film s'achève symboliquement en évoquant la scène érotique qui a lieu à la fin du roman Le Bleu du ciel, l'acte sexuel que consument les deux protagonistes, sur la terre fraîche " d'un cimetière étoilé » (Bataille 2009: 175).

Une séquence de Georges Bataille, à perte de vue résume à notre sens la conception de l'érotisme bataillien et plus particulièrement les éléments qui entrent en jeu dans l'érotisme tel que Bataille le définit. Il s'agit d'un épisode de Madame Edwarda, dont l'incipit met en scène un narrateur qui déambule seul, la nuit, dans les rues de Paris et qui se décide à entrer dans un bordel où il fait la découverte d'une prostituée, Edwarda, avec qui il a une relation charnelle. Dans la séquence de Labarthe, deux extraits de l'œuvre Madame Edwarda sont lus par la voix off et les pages sont montrées à l'écran, le premier extrait débute par ces mots :

Madame Edwarda me fascinait, je n'avais jamais vu de fille plus jolie - ni plus nue. Sans me quitter des yeux, elle prit dans un tiroir des bas de soie blanche : elle s'assit sur le lit et les passa. Le délire d'être nue la possédait : cette fois encore, elle écarta les jambes et ouvrit la fente (Bataille 2010a : 38-39).

Ce premier extrait de Madame Edwarda que Labarthe met en images intervient après le rapport sexuel entre le narrateur et Edwarda. Le deuxième extrait de Madame Edwarda que Labarthe montre est antérieur, il correspond à la rencontre entre le narrateur et la prostituée qui se présente à lui : « De mon hébétude, une voix, trop humaine, me tira. La voix de Madame Edwarda, comme son corps gracile, était obscène. Tu veux voir mes guenilles ? disait-elle» (Bataille 2010a : 33-34). Dans le texte initial, Bataille a recours à une écriture elliptique pour signifier que le rapport sexuel entre les deux protagonistes s'est produit. Labarthe reprend le texte de Bataille mais choisit au sein de sa séquence de permuter l'ordre des deux scènes de Madame Edwarda, peut-être pour désorienter le spectateur. Cette séquence reflète assez bien le traitement temporel que le réalisateur impose à son film, fait de va-et-vient incessants.

Dans la séquence de Madame Edwarda que Labarthe évoque, l'atmosphère du bordel est rendue par le verre brisé qui laisse deviner les conduites désordonnées des clients. L'érotisme est présent à travers le jeu ambigu entre ce qui est montré et ce qui est caché, entre la dignité affichée des visages des hommes et l'inconvenance dissimulée du sexe féminin qui est suggéré hors du champ. La dialectique entre ce qui est visible et ce qui ne l'est pas est essentielle à l'érotisme de Bataille et Labarthe parvient à la recréer dans cette séquence même si celle-ci s'achève finalement sur le plan d'un sexe féminin, l'œil du sexe d'Edwarda, qui fait écho à d'autres yeux, à ceux du père aveugle de l'auteur et, dans Histoire de l'œil, à l'œil de Granero, ce torero mort énucléé, ainsi qu'à l'œil du chat du premier chapitre.

Mais l'œil est aussi, et surtout, la trame d'Histoire de l'œil de Bataille et celle du récit de Labarthe au titre très évocateur, Georges Bataille, à perte de vue. Quant à l'image finale, celle du supplice chinois appelé Cent Morceaux, il s'agit du supplice de Fou Tchou Li, qui eut lieu le 10 avril 1905 et dont Georges Bataille eut connaissance grâce à 
son analyste Adrien Borel, qui donna à l'écrivain les photographies que Louis Carpeaux fit de ce supplice. Cette image hantait Bataille, lui qui était obsédé par la mutilation. Le supplice des Cent Morceaux incarne l'érotisme bataillien car, pour l'écrivain, l'érotisme se mêle à l'horreur et l'attraction et la répulsion sont au cœur du phénomène érotique, à l'image d'Edwarda qui fascine et répugne en même temps.

\section{Les éléments fantastiques}

Comme le laisse entrevoir la séquence de Madame Edwarda, l'ensemble du film de Labarthe est imprégné d'une atmosphère fantastique. Dès la deuxième minute, le réalisateur introduit un plan-séquence sur une nature déchaînée où l'orage, les éclairs, la nuit et la lune rappellent certaines scènes d'Histoire de l'œil ou certains passages de l'œuvre à laquelle Labarthe ne fait pas directement référence, Le Mort (1967). Le soleil, le plus souvent aveuglant, est un autre élément récurrent essentiel de l'imaginaire bataillien que Labarthe n'omet pas de citer dans Georges Bataille, à perte de vue.

Par ailleurs, le fantastique est suggéré grâce au mystère qui entoure la société secrète Acéphale et l'allusion faite par Labarthe au sacrifice humain qui aurait été envisagé par les membres de cette société dans la forêt de Saint-Nom-la-Bretèche. En effet, le nom 'Acéphale' n'évoque pas seulement la revue mais aussi la société ésotérique secrète que fonda Georges Bataille en 1937. L'écrivain orchestrait des cérémonies nocturnes en pleine forêt pour susciter des pratiques rituelles ; selon Michel Camus les activités d'Acéphale se basaient sur des " méditations mortifères ou rites orgiaques » (Camus 1980 : i). En faisant référence à la célébration d'un sacrifice rituel, dont les membres de la société secrète Acéphale auraient été les seuls témoins, Labarthe dote son film de mystère.

En outre, Labarthe renforce le caractère fantastique de Georges Bataille, à perte de vue grâce au personnage féminin nu, qui fait irruption dans le récit en menaçant la vraisemblance de celui-ci. La jeune femme apparait pour la première fois au milieu d'une forêt, attachée au tronc d'un arbre, peut-être pour signifier le sacrifice humain que nous venons d'évoquer. Puis elle apparaît et disparaît entre les étagères vides de la salle de lecture de la Bibliothèque d'Orléans, pour être ensuite filmée dans la réserve où étaient entreposés les ouvrages, assise sur le sol, à côté de rats qui se promènent autour d'elle, peut-être s'agit-il là d'un clin d'œil à l'œuvre de Bataille, Histoire des rats (1947a). En ayant recours à ce personnage féminin, Labarthe introduit le spectateur au cœur du fantastique tel que le conçoit Tzvetan Todorov dans son Introduction à la littérature fantastique : "Dans ce monde qui est le nôtre, celui que nous connaissons [...] se produit un événement qui ne peut s'expliquer par les lois de ce même monde familier » (Todorov 1970 : 29). L'apparition répétée de la femme nue peut être considérée comme un événement surnaturel qui a trait au fantastique.

Les éléments d'ordre fantastique qui interviennent dans Georges Bataille, à perte de vue sont nombreux, ainsi en est-il de la folie, de l'horreur ou de la nécrophilie. La figure du double, dont Tzvetan Todorov déclare qu' " il en est question dans maint texte fantastique » (Todorov $1970: 151)$, est omniprésente dans le film, le personnage 
du voyageur incarnant le double de Labarthe lui-même. De plus, le voyageur, qui suit les traces de Bataille à la recherche d'une vérité secrète sur l'auteur, et l'enquête qu'il semble mener confèrent au film son suspense, le suspense étant lui aussi un ingrédient propre au fantastique. Enfin, Georges Bataille, à perte de vue n'est pas exempt d'humour, un élément d'essence fantastique, notamment sous-jacent dans la séquence de Madame Edwarda que Labarthe met en scène en s'attardant à l'expression des visages des hommes qui fixent le sexe de la prostituée.

Dans Georges Bataille, à perte de vue, Labarthe oppose l'œil, considéré habituellement comme un organe noble, à des éléments qu'il désigne comme « la merde, la pourriture ou la puanteur ", autant d'éléments caractéristiques de l'hétérologie définie par Bataille, même si ce concept n'est pas cité par le réalisateur. L'atmosphère fantastique qui se dégage de Georges Bataille, à perte de vue repose aussi sur les tensions entre l'omniprésence de l'œil, organe de la conscience, et les nombreuses allusions à la matière basse qui traduisent la passion anti-idéaliste qui animait Bataille.

\section{Conclusion}

Bataille a longtemps été considéré comme inclassable dans le paysage intellectuel ou culturel. Poète, philosophe, essayiste, économiste ou mystique, l'auteur de L'Érotisme semble avoir échappé aux catégorisations et aux cloisonnements entre les genres. Lui qui déclarait peu avant sa mort : « Je dirais volontiers que ce dont je suis le plus fier, c'est d'avoir brouillé les cartes... » (Chapsal 1973 : 29), n'a cessé de déconcerter la critique littéraire qui lui a souvent été hostile. Bataille est rangé, la plupart du temps, dans la catégorie des penseurs mais est rarement considéré comme un romancier. Dans Georges Bataille, à perte de vue, Labarthe privilégie le Bataille romancier et poète en se penchant sur ses œuvres littéraires. En s'intéressant de près au texte littéraire bataillien, qui est souvent mythifié, Labarthe parvient à redonner à Bataille sa littérarité.

En effet, dans son film Georges Bataille, à perte de vue Labarthe rend hommage à un auteur souvent mal lu et caricaturé et il prône une réhabilitation du Bataille auteur d'œuvres littéraires, de textes auxquels le film donne vie. En mettant en images les récits les plus emblématiques de Bataille, Labarthe confère à son film une intensité dramatique et une force émotionnelle étonnantes. Par ailleurs, le portrait que Labarthe dresse de Bataille opère un glissement de la monstration à la narration poétique, un cheminement a ainsi lieu entre les premières images du film, les couloirs de Radio France, et la forêt auréolée de mystère, le décor de la fin ouverte que propose le film.

Dans le but de revendiquer la littérarité propre à l'œuvre batallienne, Labarthe déploie dans son film maints dispositifs narratifs qui ne visent pas à enrichir le récit par la fictionnalité mais à recréer la singularité de l'écrivain en soulignant la puissance, souvent subversive, de récits inédits dans le paysage de la littérature française du $\mathrm{XX}^{\mathrm{e}}$ siècle. En ayant recours à un dispositif narratif résolument littéraire et en multipliant les stratégies de narrativisation, Labarthe devient écrivain de Bataille et livre une vérité sur l'auteur, sur ce poète de l'extrême, une vérité qui se situe au-delà des mots et des images, à perte de vue. 


\section{BIBLIOGRAPHIE}

BATAILLE, Georges (1944): Le Coupable. Paris: Gallimard. (1947a): Histoire des rats. Paris: Édition de Minuit. (1947b) : Méthode de méditation. Paris: Éditions Fontaine.

(1970a): Cuvres complètes, Tome I. Paris: Gallimard.

(1970b): Cuvres complètes, Tome II. Paris: Gallimard.

(1973): CEuvres complètes, Tome V. Paris: Gallimard. (2001a): Histoire de l'œil (1928). Paris: Gallimard. (2001b): L’Érotisme (1957). Paris: Éditions de Minuit. (2009): Le bleu du ciel (1957). Paris: Éditions 10/18. (2010a): Madame Edwarda (1941). Paris: Éditions 10/18. (2010b): Le Mort (1967). Paris: Éditions 10/18.

BESNIER, Jean-Michel (1998): Éloge de l'irrespect et autres écrits sur Georges Bataille. Paris: Descartes \& Cie.

BRETON, André (1929): Second manifeste du Surréalisme. Paris: Gallimard.

CAMUS, Michel (1980): "L'acéphalité ou la religion de la mort". Acéphale, religion sociologie - philosophie, 1936-1939. Paris: Jean-Michel Place.

CHAPSAL, Madeleine (1973): "Georges Bataille", Les écrivains en personne. Paris: René Julliard, Union Générale d'Éditions: 21-33.

CORNILLE, Jean-Louis (2004): Bataille conservateur. Emprunts intimes d'un bibliothécaire. Paris: L'Harmattan.

HOLLIER, Denis (1974): La prise de la Concorde. Essais sur Georges Bataille. Paris: Gallimard.

LABARTHE, André S. (1997): Georges Bataille à perte de vue. France 3: Un siècle d'écrivains.

LEIRIS, Michel (1963): "De Bataille l'impossible à l'impossible Documents", Critique. $\mathrm{N}^{\circ}$ 195-196: 685-693.

(1988): À propos de Georges Bataille. Paris: Fourbis.

MAYNÉ, Gilles (2003): Georges Bataille, l'érotisme et l'écriture. Paris: Descartes \& Cie.

PIEL, Jean (1982): "L'homme Bataille", La Rencontre et la Différence. Paris: Fayard: 123-147.

RUEDA, Amanda (2014): "Du portrait cinématographique documentaire au portrait en sciences de l'information et de la communication", Sciences de la société. Vol. 92: 177-191.

SICHÈRE, Bernard (2006): Pour Bataille. Etre, chance, souveraineté. Paris: Gallimard. SOUVARINE, Boris (1983): "Prologue", La Critique sociale. Paris: Éditions de la Différence.

SURYA, Michel (1992): Georges Bataille, la mort à l'xuvre. Paris: Gallimard. (1997): "Prologue", dans Bataille, Georges: Choix de lettres (1917-1962). Paris: Gallimard. 
Des récits de Bataille aux images cinématographiques : la dimension littéraire du film Georges Bataille, à perte de vue de Labarthe

(2010): "Présentation", dans Bataille, Georges: Discussion sur le péché. Paris: Nouvelles éditions lignes.

TODOROV, Tzvetan (1970): Introduction à la littérature fantastique. Paris: Édition du Seuil.

\section{PERFIL ACADÉMICO Y PROFESIONAL}

Après des études à l'Université François Rabelais de Tours, Mathilde Tremblais a obtenu un Doctorat à l'Université du Pays basque (UPV-EHU). Sa thèse internationale, soutenue en 2016 au sein du Département de Philologie Française de cette université, porte sur les expressions du moi féminin dans la littérature intime contemporaine de langue française. Mathilde Tremblais a participé à de nombreux colloques internationaux et elle est l'auteure d'une vingtaine de textes qui traitent de questions touchant aux études de genre, aux représentations du corps androgyne, aux voix féminines dissidentes ou à la littérature érotique contemporaine, autant de domaines dans lesquels elle poursuit actuellement ses recherches.

Fecha de recepción: 30/04/2020

Fecha de aceptación: 28/06/2020 